\title{
Comparative expression of matrix metalloproteinases in low-grade mucoepidermoid carcinoma and typical lung cancer
}

\author{
JIANG FAN $^{1^{*}}$, FENG-YING WU ${ }^{2 *}$, LEI WANG ${ }^{3 *}$, GE-NING JIANG ${ }^{1}$ and WEN GAO ${ }^{1}$ \\ Departments of ${ }^{1}$ Thoracic Surgery, and ${ }^{2}$ Medical Oncology, Shanghai Pulmonary Hospital, \\ Tongji University School of Medicine, Shanghai 200433; ${ }^{3}$ Department of Surgical Oncology, \\ Shandong Tumor Hospital, Shandong Academy of Medical Science, Shandong 250062, P.R. China
}

Received March 3, 2011; Accepted July 27, 2011

DOI: $10.3892 / \mathrm{ol} .2011 .387$

\begin{abstract}
The molecular profile of low-grade mucoepidermoid carcinomas remains to be clarified. In the present study, matrix metalloproteinase (MMP) expression was compared in low-grade mucoepidermoid carcinoma (MEC) and typical lung cancer. The expression of MMP-2, MMP-7 and MMP-9 was detected by immunohistochemistry in a cohort of 110 patients (34 with low-grade MEC and 76 with matched typical lung cancers). A positive MMP-2 expression was found to be 35.29 vs. $65.79 \%$ in low-grade MEC and typical NSCLCs ( $\mathrm{p}=0.003$ ); a positive MMP-7 expression was 41.18 vs. $55.26 \%(\mathrm{p}=0.172)$; and a positive MMP-9 expression was 35.29 vs. $57.89 \%(\mathrm{p}=0.028)$. In conclusion, the expression of MMP-2 and MMP-9 in low-grade MEC is lower than that in typical lung carcinomas.
\end{abstract}

\section{Introduction}

Primary salivary-type lung tumors are rare intrathoracic malignancies and account for approximately $0.1-0.2 \%$ of all lung cancers $(1,2)$. These tumors used to be grouped under the name 'bronchial adenoma', a term that was used to describe a group of slow-growing neoplasms thought to arise from the bronchial glands, and included adenoid cystic carcinomas (ACC), mucoepidermoid carcinoma (MEC), mixed tumors and carcinoid tumors $(1,3)$. Intrathoracic MEC tumors are usually considered to be mild-behavior malignancies and patients have significantly better outcomes than typical non-small-cell lung cancer. MECs are classified as low- or high-grade depending on the number of mitoses present and the levels of necrosis and nuclear pleomorphisms. Low-grade

Correspondence to: Dr Ge-Ning Jiang, Department of Thoracic Surgery, Shanghai Pulmonary Hospital, 507 Zheng Min Rd, Yangpu, Shanghai 200433, P.R. China

E-mail: geningjiang@yahoo.com

${ }^{*}$ Contributed equally

Key words: non-small cell lung cancer, matrix metalloproteinases, mucoepidermoid carcinoma tumors tend to contain a higher proportion of mucous cells and high-grade tumors contain more squamous cells (1). The outcomes of patients with low-grade MEC were more positive than those of patients with typical lung carcinoma $(1,3-6)$. These types of tumor have a lower tumor grade, lower clinical stages and patients have more complete surgical procedures compared with other types of lung cancer, which partially explains the favorable prognosis. However, the molecular characteristics of MEC should be further investigated.

Basement membranes (BMs) line organs and smaller structures, such as the epithelium, capillary walls, alveoli and the pleura. Degradation of the BM is required for local invasion of the tumor cells and further degradation is required in metastasis (7). In carcinogenesis, the cancer cells gain the ability to degrade extracellular components, including the $\mathrm{BM}$, by producing proteolytic enzymes. Matrix metalloproteinases (MMPs) are enzymes that degrade various sections of BMs. These enzymes are associated with the ability of cancer cells to penetrate into connective tissues. Approximately 23 different MMPs have been identified thus far. Among them, MMP-2 and MMP-9 are most often associated with various malignancies. In various carcinomas, MMPs have been associated with poor prognosis, invasiveness or poor differentiation (7-9). Furthermore, lung cancer cells produce MMP-2 and MMP-9 in vitro (8). MMP-2 was found to be correlated to tumor spread in NSCLC (10). In adenocarcinoma of the lung, patients with a positive immunoreactivity for MMP-9 or MMP-2 have also been found to have an unfavorable prognosis when compared to patients with a lack of immunoreaction for MMP-9 or MMP-2 (9). In early-stage NSCLC, strong MMP-2 immunoreactivity predicts an unfavorable outcome (7).

Due to the low morbidity rate of MECs, the molecular profiles of this disease were not well addressed. Consequently, for the first time, the expression of MMP-2, MMP-7 and MMP-9 was compared in 34 patients with low-grade MECs to that of 76 matched NSCLC patients.

\section{Materials and methods}

Study population. In total, 37 cases of MEC were diagnosed and treated with complete surgery in the Shanghai Pulmonary Hospital between 2000 and 2008. MEC tumors were divided into high- or low-grade by two experienced pathologists on the 


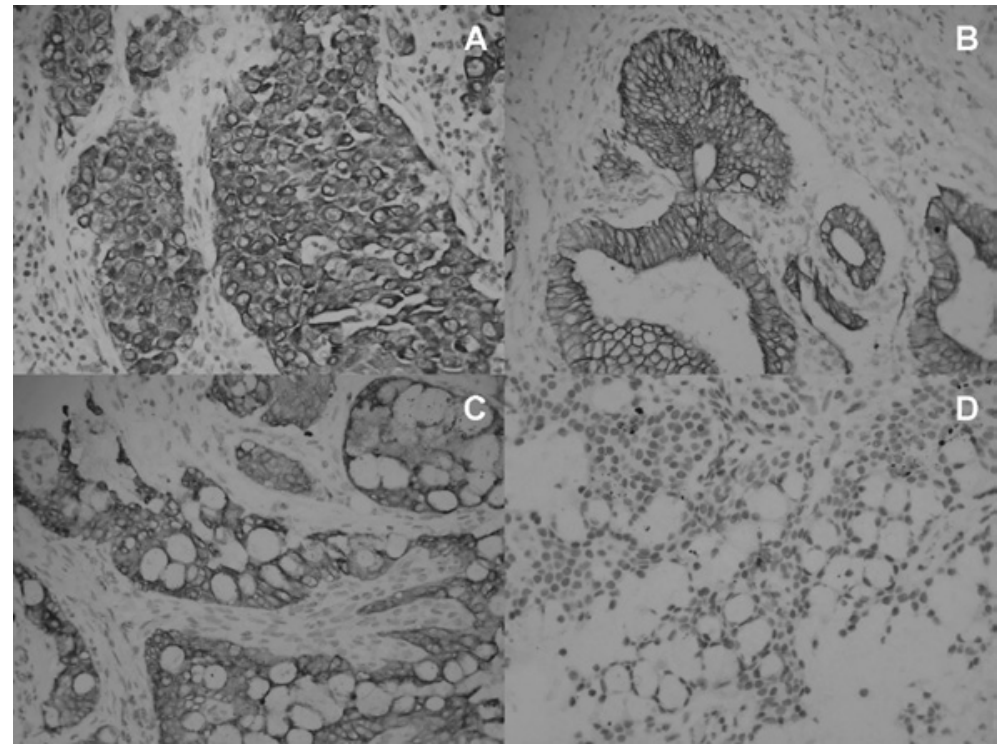

Figure 1. Immunohistochemical results of positive MMPs. (A) Immunohistochemical results of positive MMP-2 expression in a typical lung cancer specimen. MMP-2 was expressed in well-differentiated squamous cell carcinoma. MMP-2 was selectively located in the cell membrane and cytoplasm of tumor cells, but not in normal inflammatory cells or stromal cells (original magnification, x400). (B) Immunohistochemical results of the positive MMP-7 expression in a typical lung cancer specimen. MMP-7 was diffusely expressed in adenocarcinoma cells. MMP-7 was selectively stained in the majority of the tumor cells, but not in normal inflammatory cells or stromal cells (original magnification, x400). (C) Immunohistochemical results of the positive MMP-9 expression in a lowgrade MEC specimen. MMP-9 was diffusely expressed in most of the low-grade MEC cells (original magnification, x400). (D) Immunohistochemical results of the negative MMP-9 expression in a low-grade mucoepidermoid carcinoma specimen. MMP-9 was diffusely expressed in only a few of the low-grade MEC cells (original magnification, $\mathrm{x} 400$ ).

basis of mitotic activity and cellular differentiation (6,11-12). A total of 76 consecutive typical NSCLC patients with cancers at the same stage were selected as a control cohort during the same period. A preoperative staging computed tomographic (CT) scan was performed for each patient. The histology of each specimen was assessed according to the WHO classification and the pathological stage of the staging system. The exact TNM classification (UICC, 2002) and the stage of the tumor were recorded by reviewing the clinical, radiological and histopathological statements from the patient files. Histologically, the low-grade variant has a relatively benign course allowing for conservative pulmonary resection as the sole therapy (13). The typical lung cancer patients and the high-grade MEC patients received chemotherapy, while the low-grade MEC patients received surgery. No patient received either chemotherapy or radiotherapy prior to surgery. The experimental protocols were approved by the appropriate institutional review committee and met the guidelines of the responsible governmental agency.

Immunohistochemistry. MMP-2 mouse monoclonal antibody clone 42-5D11 recognizing latent and active MMP-2, at a dilution of 1:50 (Chemicon International Ltd., Billerica, MA, USA); MMP-7 mouse monoclonal antibody clone 141-7B2 recognizing latent and active MMP-2, at a dilution of 1:800 (Chemicon International Ltd.); and MMP-9 mouse monoclonal antibody clone 56-2A4 recognizing latent and active MMP-9, at a dilution of 1:100 (Chemicon International Ltd.), were used. Formalin-fixed paraffin-embedded sections of tissue blocks $(4-\mu \mathrm{m})$ obtained from the tumor periphery were mounted on silane-coated slides. Sections were de-waxed in xylene and rehydrated through the use of graded alcohols.
Antigen retrieval for MMP-2, MMP-7 and MMP-9 was achieved by pressure cooking slides for $2 \mathrm{~min}$ in $10 \mathrm{mM}$ citric acid buffer at $\mathrm{pH} 6$. Endogenous peroxidase activity was blocked by treatment with $2 \%$ hydrogen peroxide for $30 \mathrm{~min}$. Sections were rinsed in deionised water and then in Tris-buffered saline (TBS) containing $0.1 \%$ bovine serum albumin (BSA). To block non-specific staining, slides were incubated in $20 \%$ normal rabbit serum for $10 \mathrm{~min}$. Sections were incubated overnight at $4^{\circ} \mathrm{C}$ with the primary antibody. Sections were washed in TBS, then incubated sequentially with biotinylated rabbit anti-mouse $\operatorname{IgG}$ (Dako) at a dilution of 1:400, followed by streptavidin combined in vitro with biotinylated horseradish peroxidase at a dilution of 1:1000 (Dako). The reaction product was developed using diaminobenzidine tetrahydrochloride. Sections were counterstained with hematoxylin, then dehydrated through graded alcohols and mounted in a resinous mountant. A negative control was included for each section where the primary antibody step was deleted and the serum was left on. In addition, a positive control preparation tissue that contains the specific antigen was carried through with every batch of immunostaining to confirm that reagents were functional and to allow assessment of staining between the batches. For a number of antibodies the tissue also contained an internal positive control (Fig. 1).

Evaluation of immunohistochemistry. The extent and pattern of reactivity for MMP-2, MMP-7 and MMP-9 was recorded by two independent observers in a blind manner, using objectives with $\times 10$ and $\times 40$ magnification. The extent of expression was scored as 0 for no staining, $<20 \%, 20-50 \%$ and $51-100 \%$. A similar semiquantitative scale of $0,+,++$, or +++ was used to assess the intensity of staining in comparison with a 
Table I. Baseline characteristics of the two groups of patients.

\begin{tabular}{|c|c|c|c|c|c|}
\hline & $\operatorname{MEC}(n=34)$ & Prevalence & Typical NSCLC (n=76) & Prevalence & P-value \\
\hline \multicolumn{6}{|l|}{ Gender } \\
\hline Male & 20 & $58.8 \%$ & 58 & $76.3 \%$ & \\
\hline Female & 14 & $41.2 \%$ & 18 & $23.7 \%$ & \\
\hline \multicolumn{6}{|l|}{ Age } \\
\hline Mean & 35.94 & - & 63 & - & \\
\hline Median & 30 & & 65.5 & & \\
\hline Range & - & $13-73$ & - & $43-76$ & \\
\hline Stage & & & & & 0.589 \\
\hline $\mathrm{Ib}$ & 16 & $47.1 \%$ & 40 & $52.6 \%$ & \\
\hline $\mathrm{IIb}$ & 18 & $52.9 \%$ & 36 & $47.4 \%$ & \\
\hline
\end{tabular}

MEC, mucoepidermoid carcinoma; NSCLC, non-small lung cancer.

Table II. The staining of MMPs in two groups of patients.

\begin{tabular}{ccccc}
\hline & Type & Negative & Positive & Prevalence \\
\hline MMP-2 & & & 0.003 \\
& Low-grade MEC & 22 & 12 & $35.29 \%$ \\
Typical NSCLC & 26 & 50 & $65.79 \%$ \\
MMP-7 & & & $41.18 \%$ \\
& Low-grade MEC & 20 & 14 & $55.26 \%$ \\
MMP-9 & Typical NSCLC & 34 & 42 & $35.29 \%$ \\
& Low-grade MEC & & 12 & 5.028 \\
& Typical NSCLC & 22 & 44 & $57.89 \%$ \\
\hline
\end{tabular}

MEC, mucoepidermoid carcinoma; NSCLC, non-small lung cancer; MMP, matrix metalloproteinases.

known positive control. Cases were defined as positive if the staining intensity was ++ or +++ in over at least $20 \%$ of the tumors (14).

Statistical analysis. Statistical analysis was performed using the SPSS software system (SPSS for Windows, version 11.0). The Chi-square test was used to analyze the difference between the groups.

\section{Results}

Patient characteristics. Among the 37 MEC patients, 34 cases were diagnosed as low-grade and only 3 cases were diagnosed as high-grade. The characteristics of low-grade MEC patients and typical NSCLC patients included in the study are shown in Table I. Low-grade MEC patients comprised 20 males with a mean age of 35.94 (SD 7.54) years (median 30, range 13-73) with stage Ib-IIb. For the three cases of high-grade MEC, two cases were found with a high expression of all three types of MMPs, and for another case, only MMP-2 was detected with a high expression. Due to the small number of high-grade cases, the expression of MMPs was compared only in low-grade MEC and typical NSCLC.

Expression of MMP-2 and MMP-9 is significantly lower in low-grade MEC than typical NSCLC. To investigate the potential relationship between MMP expression and low-grade MEC, we compared the expression levels of MMPs in low-grade MEC with those in typical NSCLC. The expression of MMP-2 and MMP-9 was found to be significantly different in the two groups; the MMP-2 positive percentage was 35.29 (12 of 34 cases) in low-grade MEC, 65.79 (50 of 76 cases) in typical NSCLC ( $\mathrm{p}=0.003)$, and the MMP-9 positive percentage was 35.29 (12 of 34 cases) in low-grade MEC and 57.89 (44 of 76 cases) in normal NSCLC, respectively $(p=0.028)$ (Table II). Thus, the results show the expression levels of MMP-2 and MMP-9 were found to be lower in low-grade MEC patients than in typical NSCLC patients.

Expression of MMP-7 shows no difference in low-grade $M E C$ and typical NSCLC. No significant difference was found for MMP-7 expression between low-grade MEC and typical 
NSCLC tissues. The positive MMP-7 percentage was 41.18 (14 of 34 cases) in low-grade MEC and 55.26 (42 of 76 cases) in typical NSCLC individually ( $\mathrm{p}=0.172)$.

\section{Discussion}

MEC tumors are usually considered to be low-grade malignancies and patients have significantly better outcomes than typical non-small-cell lung cancer patients. Molina et al (1) reported that the overall 5- and 10-year survival rates for MEC were $88 \%$. Patient outcomes with MEC were found to be better than those of patients with typical lung carcinoma (13). Several studies have also reported high mortality in patients with MEC (3-6). It was demonstrated that patients with MEC, particularly those with low-grade tumors, had a better outcome (12). Another characteristic of MEC tumors is the rare metastasis to regional lymph nodes and the higher stage is correlated with locally aggressive disease (15).

Metastasis is the final stage in tumor progression from a normal cell to a completely malignant one and involves a number of steps. One of the initial steps in the metastatic process involves degradation of various components of the extracellular matrix and requires the action of proteolytic enzymes, of which MMPs are among the most crucial. Reports have shown correlations between degradation of the BM by MMPs and the metastatic potential of tumor cells $(16,17)$. Various MMPs, particularly MMP-2 and MMP-9, appear to correlate with early cancer-related deaths in $\operatorname{NSCLC}(7,18)$.

For the first time, the expression of MMP-2, MMP-7 and MMP-9 has been evaluated in MEC tumors. The current study demonstrated that the expression of MMP-2 and MMP-9 was lower in low-grade MEC than in typical lung carcinoma. The expression of MMPs emphasizes the ability of the tumor to metastasize to distant positions. The two types of MMPs investigated in this study may explain the low metastasis rate of the low-grade MEC tumor, and the better survival rate of the low-grade MEC. The reason for better prognosis of low-grade MEC than that of typical lung carcinoma may be multifactorial, and it is thought that the reason for metastasis is significant. At the same time, no difference in MMP-7 expression was found between low-grade MEC and typical lung carcinoma. One reason for this observation may be the limited sample size.

MEC patients are reportedly diagnosed at a much younger age as compared to NSCLC patients, at a mean age of approximately 40 years (1). In the current study, the mean age of MEC patients was found to be much lower than that of NSCLC patients, which is consistent with previous studies (1). This study indicated a slight trend towards females in MEC, while gender predilection has also been reported in numerous studies, with a specific predilection reported for males.

Histologically, MEC tumors are divided into high- and low-grade tumors on the basis of mitotic activity and cellular differentiation. The low-grade variant has a relatively benign course allowing for conservative pulmonary resection as the sole therapy (6). By separating MEC tumors into low-grade and high-grade tumors, Yousem and Hochholzer (11) reported a 95\% survival rate in 41 patients with low-grade tumors who had a follow-up that ranged between 2 months and 272 months. However, among the 13 patients with high-grade MEC, almost 25\% experienced recurrences and succumbed to the disease. This incidence was often correlated with locally aggressive disease, with approximately $50 \%$ of patients with high-grade tumors revealing pulmonary parenchymal invasion. In our institute, between 2000 and 2008 , only three cases of MEC were diagnosed as high-grade and two of them were with a high expression of three types of MMPs; one case only had a high expression of MMP-2. Due to the small number of patients, it was not possible to explore the difference in MMP expression between high-grade and low-grade MEC tumors.

Due to the short follow-up time in this cohort (30\% of the MEC patients were diagnosed in 3 years), the multivariate survival analyses for the MEC tumor were not conducted in the current study.

In conclusion, the results show that MMP-2 and MMP-9 expression is lower in low-grade MEC than in typical NSCLC. This reduced expression may be a reason for the non-aggressive behavior of the low-grade MEC tumor, while the expression of MMP-7 was not significantly different in the two groups. In addition, it appears that high-grade MEC revealed a higher expression of MMPs than low-grade MEC, in spite of the small numbers of high-grade MEC cases studied. Although the present results help to clarify the molecular profiles of MEC, further studies are required to elucidate the molecular mechanisms of MEC, which may lead to the appropriate treatment of MEC in the future.

\section{Acknowledgements}

The study was supported in part by a grant from the Ph.D. Programs Foundation of the Ministry of Education of China (No. 200802471012). This study also was supported in part by the Natural Science Foundation of Shandong Province (2007ZRC03088). We also gratefully acknowledge the technical assistance of two pathologists: Dr Rong-Xuan Zhang and Dr Chao-Fu Wang.

\section{References}

1. Molina JR, Aubry MC, Lewis JE, et al: Primary salivary gland-type lung cancer: spectrum of clinical presentation, histopathologic and prognostic factors. Cancer 110: 2253-2259, 2007.

2. Heitmiller RF, Mathisen DJ, Ferry JA, Mark EJ and Grillo HC: Mucoepidermoid lung tumors. Ann Thorac Surg 47: 394-399, 1989.

3. Chin $\mathrm{CH}$, Huang CC, Lin MC, Chao TY and Liu SF: Prognostic factors of tracheobronchial mucoepidermoid carcinoma-15 years experience. Respirology 13: 275-280, 2008.

4. Yang KY, Chen YM, Huang MH and Perng RP: Revisit of primary malignant neoplasms of the trachea: clinical characteristics and survival analysis. Jpn J Clin Oncol 27: 305-309, 1997.

5. Lal DR, Clark I, Shalkow J, et al: Primary epithelial lung malignancies in the pediatric population. Pediatr Blood Cancer 45: 683-686, 2005.

6. Torres AM and Ryckman FC: Childhood tracheobronchial mucoepidermoid carcinoma: a case report and review of the literature. J Pediatr Surg 23: 367-370, 1988.

7. Passlick B, Sienel W, Seen-Hibler R, et al: Overexpression of matrix metalloproteinase 2 predicts unfavorable outcome in early-stage non-small cell lung cancer. Clin Cancer Res 6: 3944-3948, 2000.

8. Gonzalez N, Rodriguez N, Tripari J, et al: RT-PCR comparative study of viral load levels in the HIV positive population in Puerto Rico before and after protease inhibitor regimen implanted. Bol Asoc Med P R 90: 16-20, 1998. 
9. Kodate M, Kasai T, Hashimoto H, Yasumoto K, Iwata Y and Manabe $\mathrm{H}$ : Expression of matrix metalloproteinase (gelatinase) in T1 adenocarcinoma of the lung. Pathol Int 47: 461-469, 1997.

10. Brown PD, Bloxidge RE, Stuart NS, Gatter KC and Carmichael J: Association between expression of activated 72-kilodalton gelatinase and tumor spread in non-small-cell lung carcinoma. J Nat Cancer Inst 85: 574-578, 1993.

11. Yousem SA and Hochholzer L: Mucoepidermoid tumors of the lung. Cancer 60: 1346-1352, 1987.

12. Moran CA, Suster S and Koss MN: Primary adenoid cystic carcinoma of the lung. A clinicopathologic and immunohistochemical study of 16 cases. Cancer 73: 1390-1397, 1994.

13. Minna JD, Roth JA and Gazdar AF: Focus on lung cancer. Cancer Cell 1: 49-52, 2002.
14. Hoikkala S, Paakko P, Soini Y, Makitaro R, Kinnula V and Turpeenniemi-Hujanen T: Tissue MMP-2 and MMP-9 are better prognostic factors than serum MMP-2/TIMP-2-complex or TIMP-1 in stage I-III lung carcinoma. Cancer Lett 236: 125-132, 2006.

15. Turnbull AD, Huvos AG, Goodner JT and Foote FW, Jr: Mucoepidermoid tumors of bronchial glands. Cancer 28: 539-544, 1971.

16. Chambers AF and Matrisian LM: Changing views of the role of matrix metalloproteinases in metastasis. J Natl Cancer Inst 89: 1260-1270, 1997.

17. Westermarck J and Kahari VM: Regulation of matrix metalloproteinase expression in tumor invasion. FASEB J 13: 781-792, 1999.

18. Sienel W, Hellers J, Morresi-Hauf A, et al: Prognostic impact of matrix metalloproteinase-9 in operable non-small cell lung cancer. Int J Cancer 103: 647-651, 2003 